\title{
Automatic Tuning of Discrete Fourier Transforms Driven by Analytical Modeling
}

\author{
Basilio B. Fraguela \\ Depto. de Electrónica e Sistemas \\ Universidade da Coruña \\ A Coruña, Spain \\ basilio@udc.es
}

\author{
Yevgen Voronenko, Markus Püschel \\ Electrical and Computer Engineering \\ Carnegie Mellon University \\ Pittsburgh, PA, USA \\ \{yvoronen,pueschel\}@ece.cmu.edu
}

\begin{abstract}
Analytical models have been used to estimate optimal values for parameters such as tile sizes in the context of loop nests. However, important algorithms such as fast Fourier transforms (FFTs) present a far more complex search space consisting of many thousands of different implementations with very different complex access patterns and nesting and code structures. As a results, some of the best available FFT implementations use heuristic search based on runtime measurements. In this paper we present the first analytical model that can successfully replace the measurement in this search on modern platforms. The model includes many details of the platform's memory system including the TLBs, and, for the first time, physically addressed caches and hardware prefetching. The effect, as we show, is a dramatically reduced search time to find the best FFT without significant loss in performance. Even though our model is adapted to the FFT in this paper, its underlying structure should be applicable for a much larger set of code structures and hence is a candidate for iterative compilation.
\end{abstract}

Keywords-automatic performance tuning, discrete Fourier transform, FFT, high-performance computing, library generators, model-driven optimization, program optimization

\section{INTRODUCTION}

There is consensus that the complexity of current computers makes analytical performance modeling exceedingly difficult and possibly intractable. As a result, adaptive libraries and program generators such as ATLAS [1], FFTW [2] and Spiral [3] resort to search or iterative compilation based on runtime measurements to explore choices and tune for performance. However, models have successfully guided optimization processes whose purpose was to choose quantitative parameters such as the degree of unrolling or tile sizes [4], [5], [6]. Unfortunately, the structure of many important algorithms such as fast Fourier transforms $(\mathrm{FFTs})^{1}$ produces an optimization search space that cannot be described using a few values. Rather, FFTs are typically written as a series of small kernels of varying size that can be combined in thousands of different ways with very different nesting and access patterns. Hence, previous work on FFT modeling [7] was based on large feature sets, large training data sets, and used an FFT specific approach.

\footnotetext{
${ }^{1}$ The terminology that we use in this paper is that FFTs are algorithms to compute the discrete Fourier transform (DFT).
}

In this paper, we present an analytical performance model that aims to replace iterative feedback-driven compilation for algorithms with regular access patterns. The model takes into account details of the memory system including the TLB, branch prediction, and is compatible with SIMD instruction set extensions. Two novel contributions of this model are the inclusion of the physically-indexed nature of the lower level caches, as well as the effect of the hardware prefetchers, which can play an important role for performance as we will show.

We have integrated the model into Spiral [3] to guide the selection of optimal DFT implementations for stateof-the-art processors. Benchmarks on Intel-based platforms show that the performance of the code generated this way is comparable and sometimes even better than the code produced by iterative compilation based on runtime measurements, while requiring only a fraction of the search time. This property is particularly relevant since the size of the search space grows exponentially with the problem size if all options are considered. As a result, for large sizes the code generation time without a model can be many hours.

The rest of the paper is organized as follows. Section II discusses related work and gives background about Spiral and the DFT. Section III describes our performance modeling approach. The modeling of the memory hierarchy is particularly complex and includes the two main novel contributions; thus, Section IV describes it in additional detail. Experimental results are shown in Section V. Finally, we offer conclusions in Section VI.

\section{BACKGROUND AND RELATED WORK}

The increasing complexity of hardware platforms makes the implementation of high-performance libraries an extremely complex task. For many important domains, compilers cannot achieve the highest possible performance for several reasons including the lack of high-level algorithm knowledge (hence they cannot apply the appropriate optimizations), the lack of a precise model of the underlying hardware, and the lack of a proper strategy to navigate the usually large set of compilation choices.

The first and third problem are addressed by a number of domain-specific automation tools [8], such as adaptive 
libraries and program generators. However, these tools often use runtime performance feedback to guide the optimization process, because of the lack of a sufficiently precise model of the underlying hardware.

In this paper we develop a detailed model for CPU and cache performance that can be integrated into a highperformance compiler or a program generator. As an application, we incorporated our model into the Spiral program generator [3] to guide the search in the FFT space. We note that FFTs have rather complex access patterns, in particular after all necessary optimizations are applied (as done by Spiral automatically).

Related work. Models are particularly useful for larger problem sizes, where the performance is strongly impacted by the memory hierarchy. Several cache models have been proposed, including the Cache Miss Equations [9], the Presburger formula based approach in [10], the Stack Distances Model [11], and the Probabilistic Miss Equations [12]. All of these models support loop nests with regular access patterns similar to the ones considered in this paper. But to date these models are restricted to choose quantitative optimizations parameters rather than the entire structure of the code [4], [5]. Also, all these models assume virtually-indexed caches, which does not reflect the reality on most modern processors and hence can diminish accuracy. As we will explain in Section IV-B, this affects particularly the modeling of code that accesses data in strides of large powers of two, as, for example, is the case for FFTs. Finally, to date no model has included hardware prefetching, a very common feature in current processors.

In [7] the performance of FFTs is predicted by applying machine learning techniques to training data obtained based on a set of detailed features of the FFT recursion strategy. The work is inherently restricted to the DFT and the CooleyTukey FFT. Our model is not restricted to FFTs, and could potentially be used in a general purpose compiler. Further, it can predict performance on more complex architectures, and relies on training to a very limited extent (only to select few key numeric parameters), thus reducing the potential of overfitting.

Spiral. Spiral [3] is a program generator for linear transforms such as the DFT, discrete cosine transforms, digital filters, wavelets, and others. Spiral automates the optimization and tuning process on a given platform in two ways. First, it contains a large repository of fast transform algorithms represented in the domain-specific language SPL (Signal Processing Language), and it is able to iteratively choose and recursively combine different algorithms to find the best variant for a given transform size. Second, it automates both algorithm level and code level transformations, which are performed on SPL and lower levels of abstraction. This includes loop optimizations [13] and SIMD vectorization [14]. The choice of the algorithm and algorithm-level restructuring is driven by runtime performance feedback,

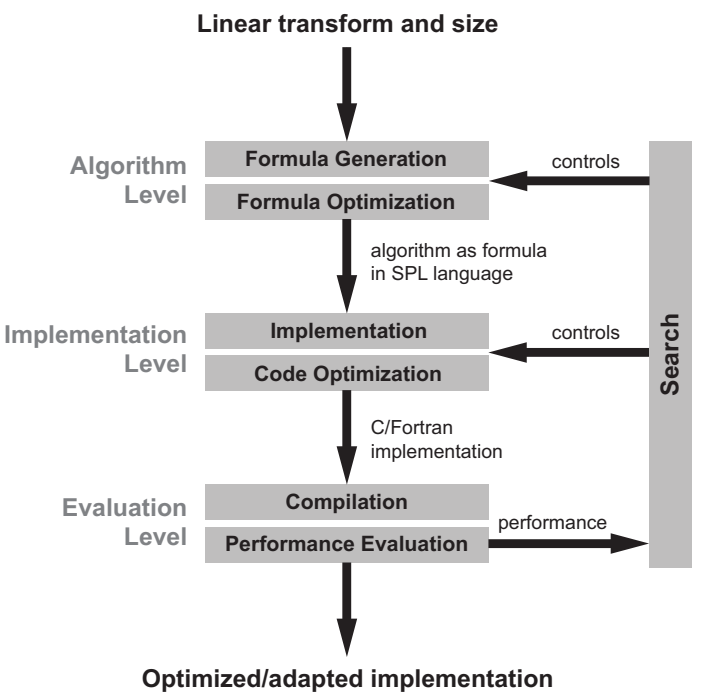

Figure 1. The program generator Spiral.

obtained by compiling and running the generated code.

Fig. 1 shows the architecture of Spiral. The input to the system is a linear transform specification and its size. The code generation is a feedback loop with three major stages (levels), which are driven by a performance metric. The "Algorithm Level" stage, generates algorithms as SPL programs (called formulas) and optimized. Next, the "Implementation Level" translates formulas into an intermediate code representation, applies traditional compiler optimizations, and outputs a program in a target language (e.g., C). Finally, the "Evaluation Level" compiles the generated programs using a standard compiler, linked with a measurement routine, and runs them to produce the performance metric, which, in turn, drives the "Search" module.

Performance model integration. We integrated our model as a performance metric into Spiral. It shortens the Spiral search time in two ways. First, the input to the model is a coarse representation of the loop structure of the final program, along with the precise information of the memory locations touched by each iteration. This information is available at the formula level, and thus the "Implementation Level" block can be omitted. Second, the performance for the feedback loop is obtained by the model rather than by compiling and exexcuting the target program.

Note that Fig 1, and this paper in general, considers the generation of transform code for fixed input sizes. Alternatively, the model could be integrated into an adaptive general input size library like FFTW [2], or a Spiral-generated adaptive library [15].

\section{PERFORMANCE MODELING}

The performance modeling approach successfully used in [11], [4], [12], [5] estimates the execution time as the sum of the time spent in the CPU and the time lost due to 


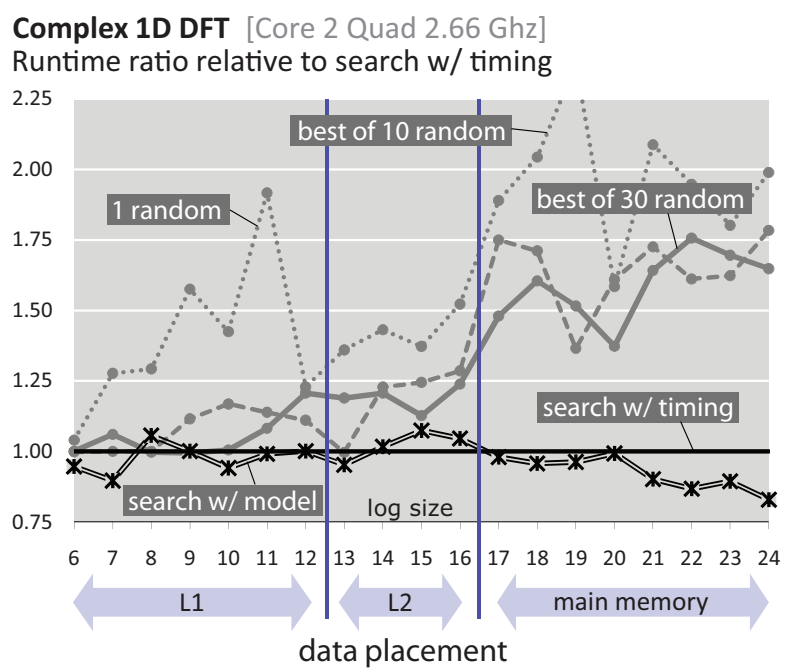

Figure 2. Runtime ratio of random implementations and implementations found using the proposed model versus the implementations found by Spiral using search with timing.

memory access stalls. This second term can be estimated as the product of the miss penalty at each cache level multiplied by the number of expected misses. Thus the estimation has the form

$$
\text { Runtime }=\text { CPUtime }+\sum_{i=1}^{N} W_{i} M_{i}
$$

in a system with $N$ levels of memory, each one of them with a miss penalty $W_{i}$, and where $M_{i}$ is the number of misses estimated for level $i$. Next, we describe our strategy to estimate the CPU time; the next section is devoted to the estimation of $M_{i}$.

For FFTs, the second term is the most important as shown in Fig. 2. The plot compares the relative difference between random and best found implementations using Spiral search with both actual timing and using model guided search (as presented in this paper). For sizes whose working set fits in the L2 cache, the miss penalties are low and CPUtime dominates the runtime. For these sizes, randomly chosen implementations perform quite well. For sizes that do not fit into the L2 cache, randomly chosen implementations perform much worse. Hence, good cache behavior prediction is crucial.

CPU modeling. Modern processors are superscalar, executing their instructions out of order and using advanced techniques such as branch prediction. The two main sources of stalls in these systems are cache misses and branch mispredictions. Thus a reasonable approach to modeling the CPU-only time of an application is to estimate the execution time of its basic blocks, and add the number of expected branch mispredictions multiplied by their associated penalty, which is typically the depth of the processor pipeline.

Also, even though we consider CPU execution time and memory stall time separately, in reality both terms overlap to some extent. This is due to the execution of instructions that are further away in the instruction stream as long as they do not depend on the data items that generate memory stalls. We model this behavior by multiplying the estimated CPU cycles by a factor between 0 (total overlap) and 1 (no overlap) before adding them to the memory stall times. In our experiments we have used a factor of 0.65 .

CPU modeling for the DFT. The DFTs generated by Spiral consist of non-perfectly nested loops whose bodies either compute kernel DFTs of sizes 2 to 32 directly, or perform buffering, which means gathering the input that is originally separated by large strides in a consecutive memory region to improve locality.

The only FFT-specific part of our model is that, based on our observations, it estimates $16 \cdot n$ CPU-only cycles for a DFT kernel of size $n$. A general model for superscalar processors such as [11] could have been used, but that would have brought little novelty, and it would have required generating the source code of the kernels. In our approach, a symbolic representation (in SPL) of the code is provided to the model, which skips Spiral's "Implementation Level" stage (Fig. 1). Buffering is memory bound. Thus, 0 cycles of CPU time are estimated for them. As for branch mispredictions, in this code they only take place in the execution of the last iteration of any loop. Thus for each execution of a whole loop we add the extra CPU time associated to a branch misprediction, unless we estimate the loop has been completely unrolled by either the compiler or even Spiral. We predict this happens whenever the loop performs less than 96 accesses to memory.

\section{MEMORY HIERARCHY MODELING}

Predicting the behavior of the memory hierarchy is difficult, since it is reacts highly sensitively to small changes in code structure, problem sizes, base addresses, cache layout and other parameters [16], [17]. Among the models available to characterize the memory hierarchy we have chosen the Probabilistic Miss Equations (PME) [12] because their probabilistic nature can accommodate the modeling of non deterministic situations such as the placement of virtual pages in physical memory, as we will see in Section IV-B. We will first explain the basics of the model, closely following [12], and then introduce the extensions developed to improve its accuracy by considering the physical indexing of the second level cache, and the hardware prefetching units.

\section{A. The Probabilistic Miss Equations}

The Probabilistic Miss Equations (PME) model [12] provides fast and accurate predictions of the memory behavior in caches with an LRU replacement policy. The model is based on the idea that cache misses take place the first time a memory line is accessed (compulsory miss) and each time a new access does not find the line in the cache because it 
has been replaced since the previous time it was accessed (interference miss). This way, the model generates a formula for each reference and loop that encloses it that classifies the accesses of the reference within the loop according to their reuse distance, that is, the portion of code executed since the latest access to each line accessed by the reference. The reuse distances are measured in terms of loop iterations and they have an associated miss probability that corresponds to the impact on the cache of the footprint of the data accessed during the execution of the code within the reuse distance. The formula estimates the number of misses generated by the reference in the loop by adding the number of accesses with each given reuse distance weighted by their associated miss probability.

The accesses that cannot exploit reuse in a loop are compulsory misses from the point of view of that loop. Still, they may enjoy reuse in outer or preceding loops, so they must be carried outwards to estimate their potential miss probability. For this reason the PME model begins the construction of its formulas in the innermost loop that contains each reference and proceeds outwards. For the same reason the PME formula for each loop is a function of the memory region accessed since the immediately preceding access to the data accessed by the reference in the loop when the execution of the loop begins. This region is called RegIn. It is used to estimate the miss probability for firsttime accesses to data within the loop. The formulas are built recursively, with the formula for each loop level being built in terms of the formula obtained for the immediately inner level. In the innermost loop containing a reference, the recursion finishes and the invocation to the formula for the inner level with a given RegIn region is substituted by the miss probability that such region generates. Those accesses for which no reuse distance has been found when the outermost loop is reached, correspond to the absolute compulsory misses, whose miss probability is one.

All the references in the DFT codes follow regular access patterns, that is, for any loop enclosing the references, they follow a constant stride with respect to the iterations of the loop. The PME formula that estimates the number of misses in loop $i$ of a reference $R$ that follows a regular access pattern as a function of RegIn, the memory region whose footprint may interfere with the reuses of the firsttime accesses during the execution of this loop, is

$$
\begin{aligned}
F_{R i}(\operatorname{RegIn})=L_{R i} & \cdot F_{R(i+1)}(\operatorname{Reg} \operatorname{In}) \\
& +\left(N_{i}-L_{R i}\right) \cdot F_{R(i+1)}\left(\operatorname{Reg}_{R i}\right),
\end{aligned}
$$

where $N_{i}$ is the number of iterations of the loop at nesting level $i$, and $L_{R i}$ is the number of different sets of lines accessed by $R$ during the execution of the loop. We can also define $L_{R i}$ as the number of iterations of the loop in which the accesses of $R$ cannot exploit reuse with respect to previous iterations of this loop. Thus this term corresponds

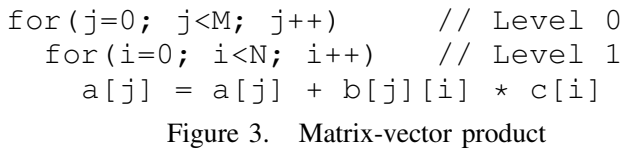

Figure 3. Matrix-vector product

to the iterations that generate first-time accesses from the point of view of this loop. The remaining $N_{i}-L_{R i}$ iterations can exploit reuse within the loop with a reuse distance of one iteration. Both terms are multiplied by $F_{R(i+1)}$, the formula that estimates the number of misses in the immediate inner loop, so as to propagate into this formula the misses generated in the inner loop. The term $\operatorname{Reg}_{R i}$ stands for the memory regions accessed during one iteration of the loop in nesting level $i$ that can interfere with the accesses of $R$ in the cache. It is used as RegIn for the iterations that can exploit reuse, since the distance for such reuse is one iteration of this loop.

$L_{R i}$ is estimated as

$$
L_{R i}=1+\left\lfloor\frac{N_{i}-1}{\max \left\{L_{\mathrm{s}} / S_{R i}, 1\right\}}\right\rfloor,
$$

where $L_{\mathrm{s}}$ is the line size, $N_{i}$ is the number of iterations of the loop, and $S_{R i}$ is the stride of $R$ with respect to loop $i$. When the index variable of loop $i$ does not index reference $R$, then $S_{R i}=0$ and $L_{R i}=1$, since the iterations of the loop do not lead the reference to access different data sets. In any other case, Equation (2) is equivalent to calculating the average number of different lines accessed during $N_{i}$ iterations with step $S_{R i}$.

Example 1. We use the matrix-vector product code in Fig. 3 to illustrate the model. We analyze the behavior of reference $b[j][i]$ to the matrix $b$ stored in row-major order, on a direct-mapped cache with 4 lines in which each line can store 2 elements of $\mathrm{b}$. We assume $M=N=4$. First, we derive a formula for the innermost loop for that reference $F_{R 1}$. Since $N_{1}=4$ and $S_{R 1}=1$, Equation (2) yields $L_{R 1}=$ 2. The resulting formula is

$$
F_{R 1}(\operatorname{Reg} \operatorname{In})=2 \cdot F_{R 2}(\operatorname{Reg} \operatorname{In})+(4-2) \cdot F_{R 2}\left(\operatorname{Reg}_{R 1}\right) .
$$

This means in two of the iterations of the innermost loop, the reference accesses new lines, since 4 elements distributed on lines of 2 elements require 2 lines, while the other 2 iterations reuse the line accessed in the immediate preceding iteration. The reuse distance for the first accesses to the lines is (yet) unknown, but the distance for the reuses is one iteration of this loop. We now derive the PME $F_{R 0}$ for the outermost loop. Since here $N_{0}=4$ and $S_{R 0}=4$, we have $L_{R 0}=4$ and

$$
F_{R 0}(\operatorname{Reg} \operatorname{In})=4 \cdot F_{R 1}(\operatorname{Reg} \operatorname{In})+(4-4) \cdot F_{R 1}\left(\operatorname{Reg}_{R 0}\right) .
$$

This means the outermost loop accesses 4 different set of lines (SOLs). When the formulas are composed, the final number of misses for reference $b[j][i]$ is

$F_{R 0}(\operatorname{Reg} \operatorname{In})=8 \cdot m i s s P r o b(\operatorname{Reg} I n)+8 \cdot m i s s \operatorname{Prob}\left(\operatorname{Reg}_{R 1}\right)$. 
If this is the first access to $\mathrm{b}$ in the program, the first accesses to lines in this code cannot exploit reuses thanks to preceding accesses; thus missProb(RegIn) $=1$. The other 8 accesses try to reuse the line accessed in the previous iteration of loop 1; thus, their miss probability depends on $\operatorname{Reg}_{R 1}$, the memory regions accessed during such iteration.

As said above, the recursion of formulas finishes in the innermost loop, where $F_{R(i+1)}$ (RegIn) returns the probability that the accesses to the memory region RegIn accessed between the consecutive accesses by $R$ to a given line turn the second one into a miss. The PME model follows three steps to calculate the miss probability associated to a given reuse distance: access pattern identification, cache impact quantification and area vectors union.

The first step identifies the access pattern followed by each reference during the reuse distance. When several references access the same memory regions, they must be merged. The main types of access patterns found in regular codes are the sequential access and the access to regions of the same size separated by a constant stride.

The second step measures the impact of each access pattern on the cache using a vector $V$ of $K+1$ probabilities called area vector $(\mathrm{AV}), K$ being the associativity of the cache. Its first element, $V_{0}$, is the ratio of sets that received $K$ or more lines from the access, while $V_{s}, 0<s \leq K$ is the ratio of sets that received $K-s$ lines. The AV associated with the accesses to each data structure is calculated separately. The method to derive the AV depends on the access pattern considered. For example, the access to $N$ regions of the same size $T$ separated by a constant stride $S$, called $S_{\mathrm{r}}(N, T, S)$, suffices to represent all the accesses found in many DFT codes. Details of the method to derive its AV for virtuallyindexed caches can be found in [12].

The previous step of the calculation of the miss probability associated to a reuse distance yields one AV per each one of the memory regions accessed during that distance. The last step of the calculation of the miss probability summarizes the effects of these AVs merging them into a global one through an union operation based on the addition of independent probabilities. Namely, the union $(\cup)$ of two AVs $V_{\mathrm{A}}$ and $V_{\mathrm{B}}$ is calculated by:

$$
\begin{aligned}
\left(V_{\mathrm{A}} \cup V_{\mathrm{B}}\right)_{0} & =\sum_{j=0}^{K}\left(V_{\mathrm{A}_{j}} \sum_{i=0}^{K-j} V_{\mathrm{B}_{i}}\right), \\
\left(V_{\mathrm{A}} \cup V_{\mathrm{B}}\right)_{i} & =\sum_{j=i}^{k} V_{\mathrm{A}_{j}} V_{\mathrm{B}_{(K+i-j)}}, \quad 0<i \leq K .
\end{aligned}
$$

The first component of the resulting $\mathrm{AV}$, which is the ratio of cache sets that received $K$ or more lines during the reuse distance, is conversely the probability a randomly chosen set received $K$ or more lines during the reuse distance. Since a line is displaced from a LRU cache when $K$ or more lines fall in its set during its reuse distance, this is the probability the reuse affected by that interference region results in a miss.

Example 2. We complete the analysis of the behav- ior of reference $b[j][i]$ in the code of Fig. 3 calculating missProb( $\left.\operatorname{Reg}_{R 1}\right)$, which was pending in Example 1. In one iteration of the innermost loop there is an access to one element of vector $a$, one of vector $c$ and one of matrix $b$. The access to $b$ cannot interfere with the reuse in the innermost loop of the line it accesses, the one being analyzed. However, the accesses to a and c can, so they are both identified as accesses to 1 element. The area vectors (AVs) that represent the impact of the accesses to a and $c$ on the considered cache, which has 4 sets of a single line that can hold 2 elements, are both $(0.25,0.75)$. That is, an access to one element involves a single line; thus 1 of 4 cache sets receive $K=1$ or more lines from the access, while the other $75 \%$ of the cache sets receive 0 lines. The impact of both accesses is then summarized applying (3) on the corresponding AVs, which yields the global AV $(0.4375,0.5625)$. Thus the miss probability associated with $\operatorname{Reg}_{R 1}$ is 0.4375 . Equation (3) computes the output AV based on the independence of the probabilities that a cache set receives the line from a or c: since each one of these probabilities is 0.25 , the probability any of them holding a randomly chosen cache set is $0.25+0.25-0.25 \cdot 0.25=0.4375$. Also, the probability none of them holds, and thus the set is empty is $(1-0.25) \cdot(1-0.25)=0.5625$.

\section{B. Modeling of physically-indexed caches}

All the models in the literature consider virtually-indexed caches, in which the addresses that index the cache are the same the compiler and the processor see. Nevertheless, many caches are physically-indexed for a number of practical reasons [18]. In these caches only the part of address within a page (the page offset) is identical to the virtual address space. When we consider addresses in different pages, the relation is broken, because each virtual page is mapped by the operating system (OS) to an unknown physical page. This leads to unintuitive behaviors in physically indexed caches. For example, a repeated access to the elements of a vector of $2 P$ words on a direct-mapped cache of $N \cdot P$ words with $N \geq 2$, only suffers cold misses in a virtually-indexed cache, since the $2 P$ consecutive words fit in the cache. Nevertheless, the OS could have mapped the virtual pages of this vector to non consecutive physical pages in memory. What is worse, these physical pages could be mapped to the same sets of the cache if it is physically-indexed. This would lead to interferences between accesses to lines of the vector located in different pages. Fig. 4 illustrates this situation for a cache of $2 P$ words and a physical mapping of the two pages of the vector that generates conflicts in the physicallyindexed cache. Many operating systems adopt cache-aware policies to allocate physical pages, seeking cache behaviors similar to those of virtually indexed caches. Nevertheless others do not, one case being Linux, which is widely used in scientific computing. It is on this kind of OS on which we center our discussion. 


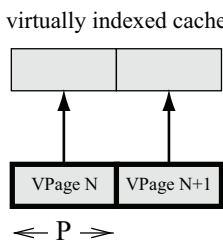

vector in virtual space

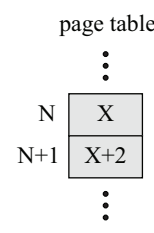

Figure 4. Example of the impact of physical indexing on cache behavior.

All the caches but the topmost ones in most processors are physically-indexed, and while their behavior is different from that of virtually-indexed caches for most codes, this is particularly true for FFTs. The reason is that FFTs access data in large power-of-two strides. Since cache ways always have a $\operatorname{size}^{2} C_{\mathrm{sk}}=C_{\mathrm{s}} / K$ that is a power of two, where $C_{\mathrm{s}}$ is the cache size and $K$ is the associativity, this means that such strides $S$ place the lines they access in at most $\max \left\{C_{\text {sk }} / S, 1\right\}$ sets in a virtually-indexed cache. This leads to systematic interferences in the sets when the number of lines per set is larger than the associativity. Nevertheless, in physically indexed caches every cache way can be divided in $C_{\mathrm{v}}=C_{\mathrm{sk}} / P$ page sets, that is, groups of cache sets that can receive data from the same page, and the lines of the access can be then distributed on $C_{\mathrm{v}} \cdot \max \{P / S, 1\}$ different sets. This number is much larger than $\max \left\{C_{\mathrm{sk}} / S, 1\right\}$ for large values of $S$, leading to fewer lines per set and thus much smaller miss rates.

The effects of physical indexing can be modeled in the PME model in the step in which an access pattern identified in the code is mapped to an area vector (AV) that represents the impact of its footprint on the cache. The access patterns found in the FFT code generated by Spiral can very often be characterized as accesses to $N$ regions of the same size $T$ separated by a constant stride $S$. We call this access pattern $S_{\mathrm{r}}(N, T, S)$. We now explain how to calculate the AV for $S_{\mathrm{r}}$ in a physically-indexed cache. Since this access pattern is a generalization of the sequential access to $n$ elements, and these are the two patterns supported by the PMEs [12], our extension allows modeling the behavior of any code analyzable by PMEs in physically indexed caches. Some Spiral-generated FFTs have access patterns not covered by $S_{\mathrm{r}}$. We omitted these since their performance was not competitive, that is, no needed in the search.

A first step in the calculation of the AV for $S_{\mathrm{r}}(N, T, S)$ in a physically-indexed cache is to simplify it if $S \leq L_{\mathrm{s}}$, the line size. In that case, since the stride is less than or equal to a cache line size, which is the granularity of placement in the cache, we can represent it as an access to a region $S_{\mathrm{r}}\left(1, T^{\prime}, T^{\prime}\right)$ with $T^{\prime}=\left\lfloor\left(N * S+L_{\mathrm{s}}-1\right) / L s\right\rfloor \cdot L_{\mathrm{s}}$. The term $L_{\mathrm{s}}-1$ is the average number of extra elements brought to

\footnotetext{
${ }^{2}$ All sizes are expressed in array elements in order to simplify the explanation.
}

the cache in the first and the last lines affected by the access, as we do not assume it to be line-aligned.

The modification to consider the indexing with physical addresses is only needed when $N \cdot S>P$, since within a length of $P$ elements or less the relation between the addresses is the same as in the virtual space, and even if the $N \cdot S \leq P$ elements are split between 2 pages, no new interferences can appear between them that the PME model for virtually-indexed caches does not consider. Also, if $C_{\text {sk }} \leq P$ the behavior of the physically and the virtuallyindexed caches are identical, thus this modification is only of interest for $P>C_{\mathrm{sk}}$, the most usual situation.

The computation of the AV in a physically-indexed cache for $N \cdot S>P$ follows a different procedure depending on whether $S \leq P$ or not. Before explaining it, let us remember that an $\mathrm{AV} V$ is a vector of $K+1$ probabilities, where $K$ is the associativity of the cache. $V_{0}$ stands for the ratio of cache sets that have received $K$ or more lines from the access pattern, and it is also conversely the probability a randomly chosen set from the cache received $K$ or more lines. $V_{i}, 0<i \leq K$ are the ratio of cache sets that received $K-i$ lines, and also the probability a randomly chosen set from the cache received exactly $K-i$ lines.

Cache impact quantification for $\boldsymbol{S} \leq \boldsymbol{P}$. The access $S_{\mathrm{r}}(N, T, S)$ affects $E=(N-1) \cdot S+T$ elements with $N-1$ holes of $S-T$ non-accessed elements in between each two consecutive regions accessed. The average number of consecutive pages over which these $E$ elements extends is $N_{\mathrm{P}}=(E+P-1) / P$, being the ratio of elements brought to the cache from each page $P_{\mathrm{A}}=\left(T+L_{\mathrm{s}}-1\right) / S$. The terms $P-1$ and $L_{\mathrm{s}}-1$ are the average number of elements affected because of non-page and non-cache line alignment respectively. The number of pages $X$ of this region the OS assigns to physical pages mapped to the same page set belongs to a binomial ${ }^{3} B\left(N_{\mathrm{P}}, 1 / C_{\mathrm{v}}\right)$. Thus, the area vector $V$ associated to this access pattern can be estimated as:

$$
\begin{aligned}
V_{K} & =\left(1-P_{\mathrm{A}}\right)+P_{\mathrm{A}} \cdot P(X=0), \\
V_{i} & =P_{\mathrm{A}} \cdot P(X=K-i), 0<i<K, \\
V_{0} & =P_{\mathrm{A}} \cdot P(X \geq K) .
\end{aligned}
$$

Cache impact quantification for $S>P$. When the stride $S$ between consecutive regions is larger than the page size $P$, each region of $T$ elements extends on two types of pages: $F$ completely filled by the region ( $F$ can be 0$)$, and at most, one at the beginning and another one at the end that are only partially filled. The average number of elements found in those starting and ending pages of each region can be computed as $R=(T-1) \bmod P+1$. As a consequence, $F=\lfloor(T-R) / P\rfloor$. Since the elements in the starting and

\footnotetext{
${ }^{3}$ Typically, $N_{\mathrm{P}}$ will not be an integer. We define the binomial distribution on a non integer number of elements $n$ as $P(X=x), X \in B(n, p)=$ $(P(X=x), X \in B(\lfloor n\rfloor, p))(1-(n-\lfloor n\rfloor))+(P(X=x), X \in$ $B(\lceil n\rceil, p))(n-\lfloor n\rfloor)$.
} 
ending page of the same region cannot overlap in the same page set, we model a region as $F$ full pages, plus another page in which only $R$ elements are accessed. The average ratio of elements brought to a page set due to the access to the $R$ remaining elements outside the full pages is estimated as $P_{\mathrm{A}}=\min \left\{1,\left(R+L_{\mathrm{s}}-1\right) / P\right\}$.

While full pages can bring data to any position in a page set, the starting and ending page can together only bring data to $P_{\mathrm{A}} \cdot P$ positions of each page set. As a result in $\left(1-P_{\mathrm{A}}\right) \cdot P$ positions, the number of lines that could be mapped to them belongs to a binomial $B\left(N \cdot F, 1 / C_{\mathrm{v}}\right)$, since only $F$ pages from each one of the $N$ regions can bring data to them. For the remaining $P_{\mathrm{A}} \cdot P$ positions, the number of lines from the considered region that could be mapped to them belongs to a binomial $B\left(N \cdot(F+1), 1 / C_{\mathrm{v}}\right)$, as also either the starting or ending page of a region can map data to them. Taking this into account, we can estimate the area vector $V$ associated to this access pattern as:

$$
\begin{aligned}
V_{i}= & P_{\mathrm{A}} \cdot P\left(X^{+}=K-i\right)+\left(1-P_{\mathrm{A}}\right) \cdot P\left(X^{-}=K-i\right), \\
& 0<i \leq K, \text { where } X^{+} \in B\left(N \cdot(F+1), 1 / C_{\mathrm{v}}\right) \\
& \text { and } X^{-} \in B\left(N \cdot F, 1 / C_{\mathrm{v}}\right), \\
V_{0}= & 1-\sum_{i=1}^{K} V_{i} .
\end{aligned}
$$

\section{Modeling hardware prefetching}

While most current architectures include hardware prefetchers, there are no attempts in the literature to model them. One of the reasons for this is that hardware vendors usually provide hints on the operation of these devices, but not data specific enough to enable their accurate modeling.

The first thing we noticed about prefetchers is that since they track the accesses to make their decision, it is mostly relevant to model them in innermost loops, where the accesses are found. Second, there is a limited number of these units and each one of them can only track one access pattern. Also, prefetchers can only be triggered when they detect accesses with a stride within a given threshold. Thus the first step to model them in a loop is to calculate the number of points accessed in each iteration with a stride larger than the threshold in the architecture considered. If this number is larger than the number of prefetchers they are considered to be unable to find any access pattern due to the excess of different accesses to track, and thus they are dismissed.

If this is not the case, the access pattern for each array is analyzed separately to detect whether the prefetcher can help it. The analysis takes into account the pattern formed by all the references to the array in the body of the innermost loop, that is, a single iteration, $S_{\mathrm{r}}\left(N_{B}, T_{B}, S_{B}\right)$, and the pattern they follow in the whole innermost loop, $S_{\mathrm{r}}\left(N_{I}, T_{I}, S_{I}\right)$. There are two situations in which the prefetcher can help. The most usual one happens when either $S_{I} \leq T h$, where $T h$ is the threshold of the prefetcher, or when $S_{B} \leq T h$ and $S_{I}=S_{B} N_{B}$. In either case the references follow small strides within a small number of pages. Since prefetchers can usually operate only within a page, which is the case in the considered architectures, they can avoid in the best case all the misses but one per page. Thus a way to model the help of the prefetcher is to consider only one access per page as a first-time access within the innermost loop. This can be done by calculating $L_{R i}$ for the formula in the innermost loop as $\left\lfloor\left(N_{I} S_{I}\right) / P\right\rfloor+1$, where $P$ is the page size, instead of applying Equation (2). Now, that approach assumes that the data brought by the prefetcher are always in the cache when the program accesses them. This actually depends on the relation between the time needed to bring data from memory to the cache, which is roughly $W$, the cost of a miss in the cache, and the cadence $C$ in CPU cycles at which the CPU accesses data from the considered stream. If $C$ were very small, the prefetcher would be useless, while if $C \geq W$ data would always arrive in time for their access. In order to take this into account, we define the ratio of effectiveness of the prefetcher as $R_{e}=\min \{C / W, 1\}$ where $C$ is the CPU time in cycles for one iteration of the considered loop, i.e, one execution of its body. Then, the PME formula for the array we are considering is computed as the weighted addition of Equation (1) calculated using $L_{R i}=\left\lfloor\left(N_{I} S_{I}\right) / P\right\rfloor+1$, with weight $R_{e}$, and the same equation with $L_{R i}$ calculated according to Equation (2), with weight $\left(1-R_{e}\right)$.

The second situation in which the prefetchers can help is when the stride $S_{I}$ with respect to the innermost loop is large, $S_{I}>S_{B} N_{B}$, but the accesses in the body of the loop fall within the threshold, i.e., $S_{B} \leq T h$. In this case the accesses are typically spread on $N_{I}$ pages, one per iteration of the innermost loop, but the accesses within each page can benefit from the prefetcher. Thus the improvement that the prefetcher can bring has nothing to do with $N_{I}$ and $S_{I}$ but with the access pattern in the body. The net effect of the prefetcher is approximately that in each one of the $N_{I}$ regions where the accesses take place following a pattern the prefetcher can detect, instead of the $L_{R i} \approx\left(N_{B} S_{B}\right) / L_{\mathrm{s}}$ potential misses that we would see if the prefetcher were not triggered, at most $1 / R_{e}$ will be seen. We model this by using $L_{R i}=\min \left\{\left(N_{B} S_{B}\right) / L_{\mathrm{s}}, 1 / R_{e}\right\}$ for the formula in the accesses in each iteration of the innermost loop.

Notice again how this modeling covers any code analyzable by PMEs and has nothing specific to FFTs.

\section{Evaluation}

We used our model to guide the search process of Spiral (Fig. 1). Specifically, we replaced the "Evaluation Level" block in Fig. 1 by our performance model. Since the model does not need the full source code, we also eliminated several stages in the "Implementation Level" block, which led to faster turnaround time for each algorithm variant.

Spiral searches over the degrees of freedom in the FFT recursion, and also over different possible restructurings of 
Table I

ARCHITECTURES TESTED. $C_{\mathrm{S}}, L_{\mathrm{S}}, K, W$ ARE CACHE SIZE, LINE SIZE, ASSOCIATIVITY AND COST OF A MiSS.

\begin{tabular}{lcccc}
\hline Architecture & L1 Parameters & L2 Parameters & TLB Parameters & \# Prefetchers \\
& $\left(C_{\mathrm{s}_{1}}, L_{\mathrm{s}_{1}}, K_{1}, W_{1}\right)$ & $\left(C_{\mathrm{s}_{2}}, L_{\mathrm{s}_{2}}, K_{2}, W_{2}\right)$ & $\left(C_{\mathrm{s}_{3}}, L_{\mathrm{s}_{3}}, K_{3}, W_{3}\right)$ & \\
\hline Pentium 4 Netburst 3GHz & $(16 \mathrm{~KB}, 64 \mathrm{~B}, 8,12)$ & $(1 \mathrm{MB}, 128 \mathrm{~B}, 8,150)$ & $(512 \mathrm{~KB}, 4 \mathrm{~KB}, 64,25)$ & 8 \\
Core 2 Quad 2.66GHz & $(32 \mathrm{~KB}, 64 \mathrm{~B}, 8,12)$ & $(4 \mathrm{MB}, 64 \mathrm{~B}, 16,125)$ & $(1 \mathrm{MB}, 4 \mathrm{~KB}, 256,15)$ & 12 \\
\hline
\end{tabular}

these algorithms to alleviate cache associativity conflicts and increase spatial locality for the memory hierarchy. We have evaluated two most commonly used search methods in Spiral: dynamic programming (DP) and the genetic search. Both methods are explained in [3].

Below, we provide two sets of experiments. In the first set we evaluate the model on a large set of random generated DFT implementations, and analyze how well the model differentiates between fast and slow programs. In the second set we compare the runtimes of the DFTs found by Spiral using true runtime measurements and using runtime estimation.

Experimental setup. For testing our model, we used the two platforms described in Table I; both ran Linux. The TLB was modeled as a cache in which each line corresponds to a virtual memory page, and the cost of a miss is the time required to update the TLB. The table also includes the number of forward prefetchers found in each architecture. We assumed a trigger threshold of 256 bytes, which means that the prefetchers begin to bring data to the $\mathrm{L} 2$ cache when two misses within a region of 256 bytes are discovered. Due to the large number of L1 misses in the DFTs and the ability of the considered architectures to parallelize misses, we used a cost of 6 cycles for the L1 miss penalty in the model.

The Spiral generated implementations were $\mathrm{C}$ programs using SSE2 double precision 2-way vector instructions through C intrinsics. All code was compiled with icc 9.1 and optimization level -O3.

The performance is reported in pseudo-GFlop/s, which is standard for DFTs. It is computed as $5 n \log _{2} n /$ (runtime [s]. $10^{-9}$ ) for an $n$ point 1D DFT, and if with $n=m^{2}$ for an $m \times m$ point 2D DFT. This metric is proportional to normalized inverse runtime.

Evaluation on random implementations. In the first set of experiments, we evaluated the model on randomly chosen DFT implementations. We generated 25,000 implementations for sizes $2^{11}-2^{19}, 17,000$ for $2^{20}$, and 2,600 for sizes $2^{21}-2^{24}$. The smaller number of implementations for the larger sizes is due to the longer time required to generate them.

Figures 5(a) and (d) show the average error rate of the model across the random implementations on Pentium 4 and Core 2. The plot includes error rates for three versions of the model: full model including prefetchers and physical indexing of L2, the full model without prefetchers, and the full model with virtual (i.e., non-physical) cache indexing. As expected, more detailed modeling reduces the error rate, with only one exception: the DFT of size $2^{24}$ on the Pentium 4, which causes memory thrashing, because the working set almost fills the memory of the machine (1 GB).

On the Pentium 4, the relatively small number of hardware prefetchers and their relative simplicity compared to those of the Core 2 reduces their importance. Nevertheless, modeling the physically-mapped nature of the L2 cache is crucial in both systems when the FFT working set does not fit in the cache.

For DFT sizes whose working sets fit in L2 cache, the error rate is larger, especially on the Core 2. Given that incache sizes have little runtime variation (see Fig. 2) this is not problematic. This inaccuracy stems to a large extent from the small size of these DFTs, which turns small absolute errors into large relative errors and does not favor the convergence of a probabilistic model like ours. For example, the DFTs of size $2^{14}$ run on average in just two and one million cycles in the Pentium 4 and the Core 2, respectively. The Core 2 error rate is larger due to its shorter running times, not modeling its L1 prefetchers, and using a fixed overlap factor between cache misses and computation, which in reality depends on the cache miss rate. The smaller miss rates in this system should lead to a better exploitation of the instruction window.

Interestingly, while modeling physical indexing proved essential to predict performance correctly for large DFTs, its influence to drive search was negligible. This is shown in Figures 5(b) and (e), which compare the best found implementations (not random) between using full and reduced models. A reason is that the patterns with many points accessed with large strides still hurt the performance of our L1 virtually-indexed caches and the TLBs, so they do not appear in fast DFTs. Modeling prefetching on the Core 2, reduces the error rate from $\approx 25 \%$ to $\approx 20 \%$ for large DFTs (a 20\% relative reduction), and improves the best found outof-cache DFT by $20 \%$ on average.

Figures 5(c) and (f) show the correlation between the predicted and the measured runtime of a set of randomly chosen implementations of $2^{20}$ point DFTs. On both platforms the absolute prediction can be quite inaccurate. For example, although the average prediction error for these DFTs on Pentium 4 is just 10\% (see Fig. 5(a)), the implementations with predicted runtime of 0.6 billion cycles in this system can actually run anywhere between 0.45 and 1.25 billion cycles. However, lack of strong correlation does not imply that the model cannot be used to drive search. In particular, both 


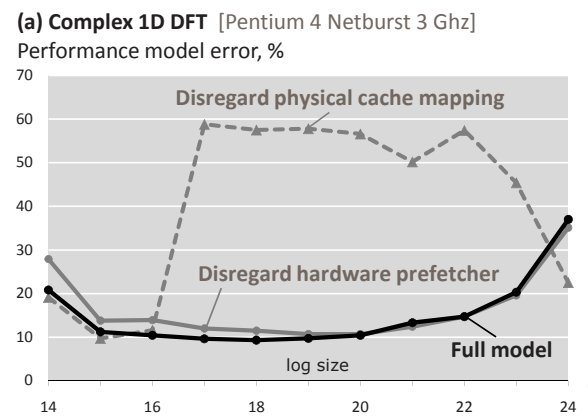

(b) Complex 1D DFT [Pentium 4 Netburst 3.0 Ghz] Performance, GFlop/s

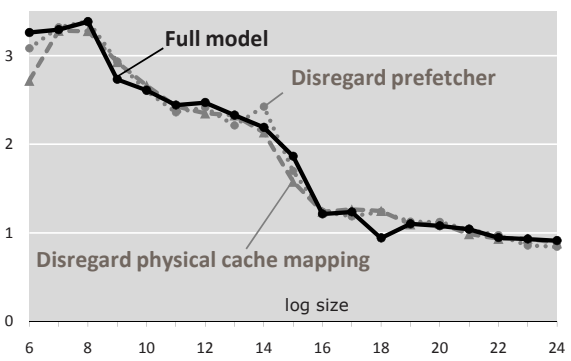

(d) Complex 1D DFT [Core 2 Quad 2.66 Ghz] Performance model error, \%

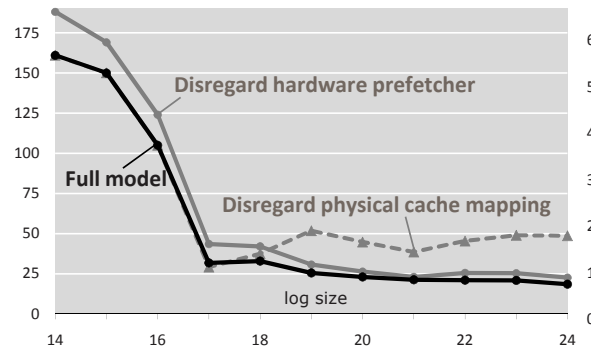

(e) Complex 1D DFT [Core 2 Quad 2.66 Ghz] Performance, GFlop/s

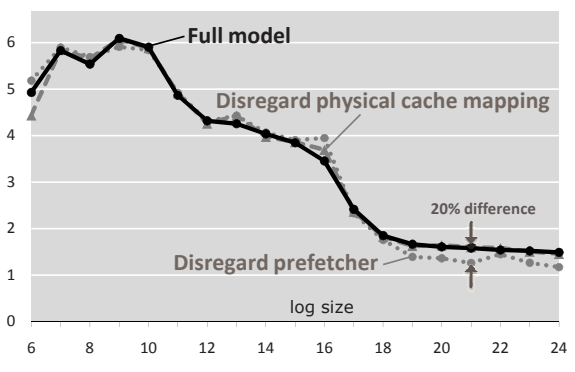

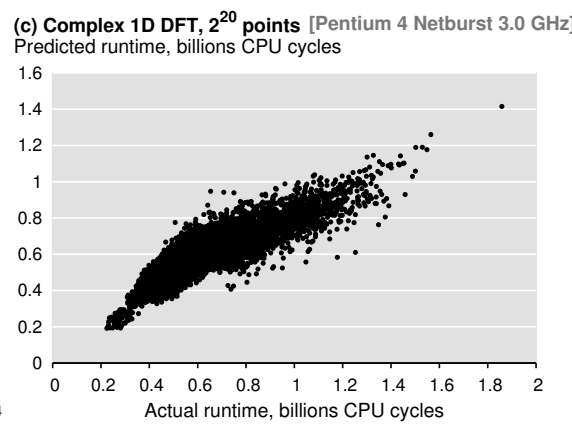

(f) Complex 1D DFT, $2^{20}$ points [Core 2 Quad $2.66 \mathrm{GHz}$ ] Predicted runtime, billions CPU cycles

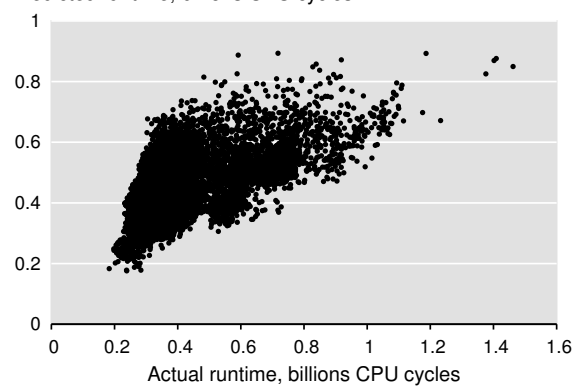

Figure 5. Performance model error for a range of DFT sizes and correlation between actual and predicted performance for the $2^{20}$ point DFT.

Figures 5(c) and (f) demonstrate that the model correctly predicts the fastest implementations. Since the objective of search is to minimize runtime, the model should still work well, and the results shown next confirm this.

Using the performance model to drive search. In the second set of experiments, we used the predicted runtime to drive Spiral's search. Figures 6(a)-(b) and (d)-(e) show the performance of the 1D and 2D DFTs found using dynamic programming and genetic search guided by both actual timing and the model. Figures 6(c) and (f) compare the respective search times. As we can see, the model-based search consistently finds implementations with a performance very close to the timed search, while reducing the search times by two orders of magnitude for large DFT sizes.

The large size FFTs found on Pentium 4 using the model are $5-10 \%$ slower than the ones found using timing. At the same time, the large size DFTs on the Core 2 are up to $10 \%$ faster. This is possible because the DP search (which worked best here), is inherently suboptimal, as it assumes context-independence of the child DFT problems.

These results show that the average error rate of the model does not tell us how well the model will drive search. The more important characteristic of the model is the ability to pick the best implementation from the space of possibilities. This can be seen from Figures 5(c) and (f), where both plots have sharp and narrow left bottom corners.

In our experiments the genetic search did not perform as well as DP with either timing or model, contrary to the results in [3]. This happens because the considered search space is now much larger due to various out-of-cache optimizations such as buffering.

\section{CONCLUSIONS}

We believe the main overall contribution of this paper is to show a successful performance model of the memory hierarchy under real world conditions. This means we considered a state-of-the-art platform and a very important and complex class of algorithms (FFTs) implemented with all necessary optimizations including SIMD vector instructions, optional buffering, and various, non-trivial loop optimizations. While the FFT was the focus here, we want to stress that little tweaking in the model was done, except for the CPU component, which only plays a minor role for out-of-cache performance. Hence, there is hope that the model can be extended to a much larger class of code types, which would make it a candidate for the iterative compilation of numeric algorithms.

\section{ACKNOWLEDGEMENT}

This material is based upon work supported by the National Science Foundation under Awards CCF 0702260 and CNS 0509432. Basilio B. Fraguela was partially supported by the Xunta de Galicia under project INCITE08PXIB105161PR and the Ministry of Education and Science of Spain, FEDER funds of the European Union (Project TIN2007-67537-C03-02). We also want to acknowledge the Centro de Supercomputación de Galicia (CESGA) for the usage of its supercomputers for this paper.

Yevgen Voronenko and Markus Püschel were supported by NSF through the awards 0325687 and 0702386 , and by 
(a) Complex 1D DFT [Pentium 4 Netburst 3 Ghz] Performance, GFlop/s

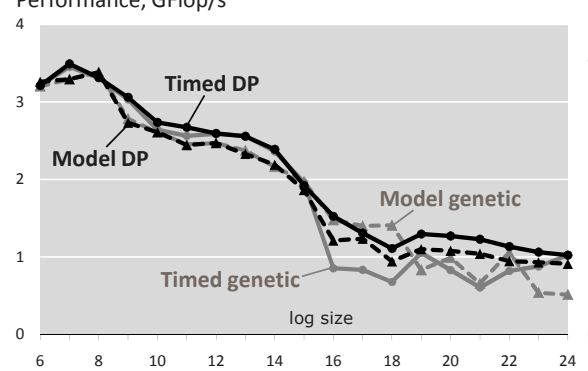

(d) Complex 1D DFT [Core 2 Quad 2.66 Ghz] Performance, GFlop/s

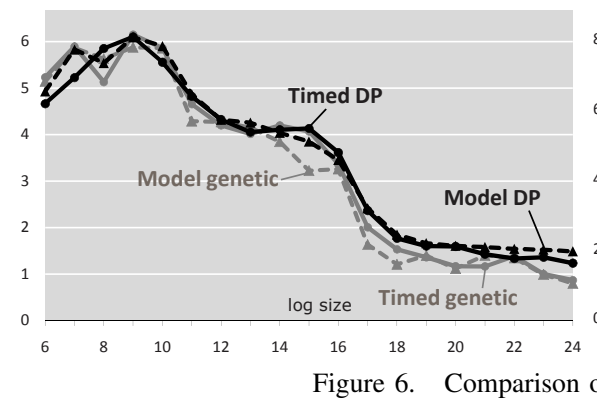

(b) Complex 2D DFT [Pentium 4 Netburst 3 Ghz] Performance, GFlop/s

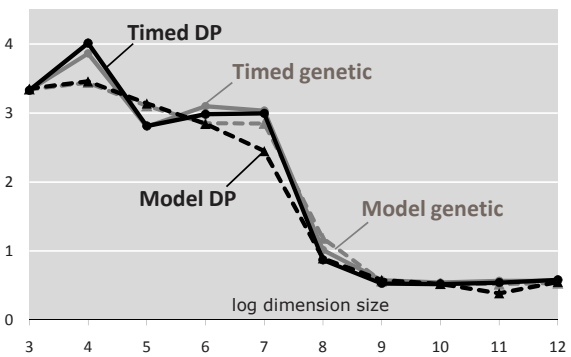

(c) Complex 1D DFT [Pentium 4 Netburst 3 Ghz] Search time, sec

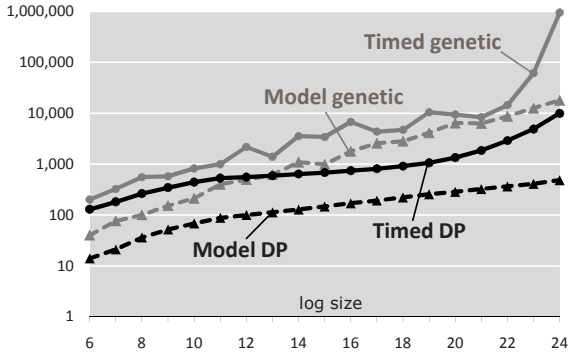

(e) Complex 2D DFT [Core 2 Quad 2.66 Ghz] Performance, GFlop/s

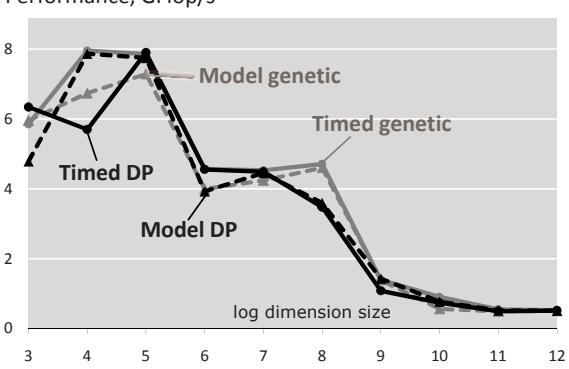

(f) Complex 1D DFT [Core 2 Quad $2.66 \mathrm{Ghz}$ ] Search time, sec

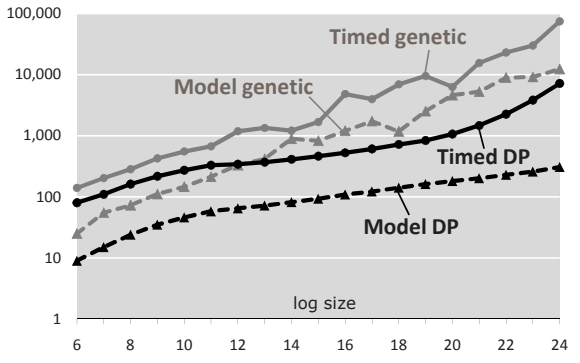

Figure 6. Comparison of performance and search times between timed and model-based search.

DARPA through the DOI grant NBCH1050009 and the ARO grant W911NF0710416, as well as by a grant from Intel.

The authors wish to extend special thanks to David Padua for his encouragement and support during the development of this research.

\section{REFERENCES}

[1] R. C. Whaley, A. Petitet, and J. Dongarra, "Automated empirical optimizations of software and the ATLAS project," Parallel Computing, vol. 27, no. 1-2, pp. 3-35, 2001.

[2] M. Frigo and S. G. Johnson, "The design and implementation of FFTW3," Proc. of the IEEE, vol. 93, no. 2, pp. 216-231, 2005.

[3] M. Püschel, J. M. F. Moura, J. Johnson, D. Padua, M. Veloso, B. Singer, J. Xiong, F. Franchetti, A. Gacic, Y. Voronenko, K. Chen, R. W. Johnson, and N. Rizzolo, "SPIRAL: Code generation for DSP transforms," Proc. of the IEEE, vol. 93, no. 2, pp. 232- 275, 2005.

[4] X. Vera, J. Abella, A. Gonzalez, and J. Llosa, "Optimizing program locality through CMEs and GAs," in Proc. Parallel Architectures and Compilation Techniques (PACT), 2003, pp. 68-78.

[5] B. B. Fraguela, M. G. Carmueja, and D. Andrade, "Optimal tile size selection guided by analytical models," in Proc. Parallel Computing (ParCo), 2005, pp. 565-572.

[6] K. Yotov, X. Li, G. Ren, M. Garzaran, D. Padua, K. Pingali, and P. Stodghill, "Is search really necessary to generate highperformance BLAS?" Proc. of the IEEE, vol. 93, no. 2, 2005.

[7] B. Singer and M. Veloso, "Learning to construct fast signal processing implementations," J. Machine Learning Research, special issue on ICML, vol. 3, pp. 887-919, 2003.

[8] J. M. F. Moura, M. Püschel, D. Padua, and J. Dongarra, "Scanning the issue: Special issue on program generation, optimization, and platform adaptation," Proc. of the IEEE, vol. 93, no. 2, pp. 211-215, 2005.
[9] S. Ghosh, M. Martonosi, and S. Malik, "Cache Miss Equations: A Compiler Framework for Analyzing and Tuning Memory Behavior," ACM Trans. Programming Languages and Systems, vol. 21, no. 4, pp. 702-745, July 1999.

[10] S. Chatterjee, E. Parker, P. J. Hanlon, and A. R. Lebeck, "Exact analysis of the cache behavior of nested loops," in Proc. Programming Language Design and Implementation (PLDI), 2001, pp. 286-297.

[11] C. Cascaval, L. D. Rose, D. A. Padua, and D. A. Reed, "Compile-time based performance prediction," in Proc. Languages and Compilers for Parallel Computing (LCPC), 1999, pp. 365-379.

[12] B. B. Fraguela, R. Doallo, and E. L. Zapata, "Probabilistic Miss Equations: Evaluating Memory Hierarchy Performance," IEEE Trans. Computers, vol. 52, no. 3, pp. 321-336, 2003.

[13] F. Franchetti, Y. Voronenko, and M. Püschel, "Formal loop merging for signal transforms," in Proc. Programming Languages Design and Implementation (PLDI), 2005, pp. 315326.

[14] — - "A rewriting system for the vectorization of signal transforms," in Proc. High Performance Computing for Computational Science (VECPAR), 2006, pp. 363-377.

[15] Y. Voronenko, F. de Mesmay, and M. Püschel, "Computer generation of general size linear transform libraries," in Proc. Code Generation and Optimization (CGO), 2009, pp. 102113.

[16] M. S. Lam, E. E. Rothberg, and M. E. Wolf, "The Cache Performance and Optimizations of Blocked Algorithms," in Proc. Architectural Support for Programming Languages and Operating Systems (ASPLOS), 1991, pp. 63-74.

[17] O. Temam, C. Fricker, and W. Jalby, "Cache Interference Phenomena," in Proc. Measurement and modeling of computer systems (SIGMETRICS), 1994, pp. 261-271.

[18] J. L. Hennessy and D. A. Patterson, Computer Architecture: A Quantitative Approach, 4th ed. Morgan Kaufmann, 2006. 